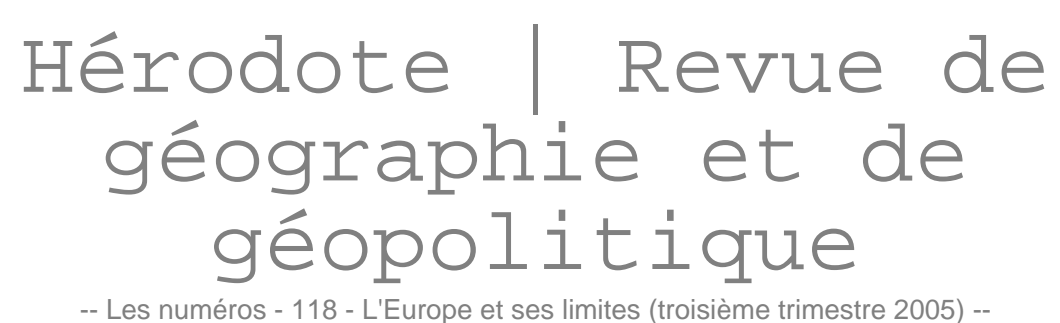

118 - L'Europe et

ses limites

(troisième

trimestre 2005)

HÉRODOTE

LEUROPE

ET SFE LIMITF

Limites futures de l'ensemble européen et soudaine restriction de l'idée européenne

Yves LACOSTE 


\section{Résumé : Limites futures de l'ensemble européen et soudaine restriction de l'idée européenne}

L'accroissement du nombre des États de l'Union européenne fut le principal argument pour souhaiter que s'établissent, par un traité constitutionnel, de nouvelles règles de fonctionnement pour I'"Europe". Ainsi ce grand ensemble économique pourrait devenir une véritable puissance politique. Ces perspectives de développement ont été brusquement stoppées en 2005 par le rejet, en France et aux Pays-Bas, sous l'effet de problèmes de politique intérieure, du projet de Constitution européenne. Cependant, parmi les causes de la crise de l'idée européenne, les explications ne manquent pas : l'opinion est mécontente de ne pas avoir été consultée lors de l'extension de l'UE et est, dans l'ensemble, hostile à l'adhésion d'un État musulman extérieur à l'Europe, la Turquie. C'est un paradoxe étonnant sinon même scandaleux que de discuter autant de l'entrée de la Turquie dans I'UE, alors que l'on ne souffle mot de la Russie, pourtant indiscutablement de culture européenne. La crise de l'idée européenne, provoquée ou révélée par les "non" français et néerlandais, reporte la question des nouveaux élargissements. II va sans doute falloir attendre plusieurs décennies pour que l'UE englobe l'ensemble des Européens, "de l'Atlantique à l'Oural" et devienne véritablement un ensemble politique. Mais cela peut aller bien plus vite si une grande menace islamiste se dressait brusquement sur sa façade sud, de la Méditerranée à l'Asie centrale, et si les Européens prenaient conscience qu'ils seront sans doute désormais seuls à devoir y faire face.

\section{Abstract : Future limits of the European entity and sudden restriction on the European idea}

The increased number of member-states in the European Union (EU) was the main reason to wish to establish new regulations in Europe with a Constitutional Treaty. Thus this great economical entity could become a real political power. These perspectives for development were suddenly stopped in 2005 by the opposition of France and the Netherlands to the European Constitution due to internal political problems. However, among the causes of the European idea crisis, explanations are numerous : the public opinion is unhappy not to have been consulted on the expansion of the EU and is, as a whole, hostile to the entry of Turkey, a Muslim state, in Europe. It is an astonishing paradox, if not scandalous, to discuss so much Turkey's entry to the EU when not a word is said about Russia, which is without a doubt of European culture. The crisis of the European idea provoked or revealed by the "No" of France and the Netherlands, delays the question of new entries. We will probably have to wait several decades before the EU encompasses all Europeans, "from the Atlantic to the Oural" and really becomes a political entity. But it could all go faster if an Islamist menace would appear in the south, from the Mediterranean to Central Asia, and if the Europeans realize that they probably would have to face it alone. 


\section{Article Complet}

Lorsqu'au milieu de l'année 2004, ce numéro d'Hérodote a été mis en chantier, il était surtout question dans la presse des nouveaux et probables élargissements de l'Union européenne (UE) : non seulement la Bulgarie et la Roumanie devaient prochainement s'ajouter à l'Europe des Vingt-Cinq, mais il était de plus en plus question des Turcs. Cela suscitait déjà diverses réserves dans l'opinion, d'autant que le président George W. Bush recommandait chaudement aux Européens la candidature de la Turquie, en tant que membre important de l'OTAN depuis plus de quarante ans. En janvier 2005, le succès de la "révolution orange" à Kiev laissait augurer la prochaine candidature de l'Ukraine à l'Europe.

Cet accroissement considérable du nombre des États de l'UE (les Quinze de 1992 étant devenus Vingt-Cinq et pourquoi pas une trentaine ?) fut le principal argument pour souhaiter que s'établissent entre eux, par un traité constitutionnel, de nouvelles règles de fonctionnement, en tenant mieux compte de leurs poids démographiques, et pour que l'"Europe" se dote d'un président et d'un ministre des Affaires étrangères. Ainsi le grand ensemble économique qu'elle est statistiquement au plan mondial (9 000 milliards d'euros de PNB) pourrait devenir une véritable puissance politique. C'était, disait-on, du moins en France, le moyen de faire face à la formidable montée en puissance économique de la Chine et de peser davantage en regard des États-Unis.

Pourtant au printemps 2003, fait géopolitique révélateur, la France et l'Allemagne, qui s'opposaient à la volonté du président américain d'aller de nouveau faire la guerre en Irak, avaient été soudain désavouées par la majorité des chefs d'États de l'UE (notamment ceux des États nouvellement adhérents d'Europe de l'Est). Ceux-ci décidèrent de suivre le Premier ministre britannique dans l'aventure irakienne aux côtés de l'armée des États-Unis. Cela prouvait que l'UE, dans cette grave épreuve de force, était encore loin de former un ensemble géopolitique. Celle-ci n'en fut que davantage objet de louanges de la part des hommes politiques et des intellectuels européens : outre sa réussite économique, n'était-elle pas porteuse, pour l'ensemble du monde, des valeurs de la démocratie ? N'était-elle pas, à la différence de l'Amérique, la "force tranquille", la "force de paix" ? Aussi l'idée d'une armée européenne paraissait-elle tout à fait superflue. L'OTAN suffisait largement, et d'ailleurs depuis la fin de la "guerre froide" quelle était son utilité ?

Ces perspectives de développement de l'UE et l'idée que l'on se faisait jusqu'alors de celle-ci ont été brusquement changées fin mai et début juin 2005, sous l'effet de problèmes de politique intérieure propres à la France et assez différemment aux Pays-Bas. En effet, le rejet du projet de "Constitution" européenne qui était soumis à référendum dans ces deux pays a immédiatement entraîné de grandes conséquences dans tous les pays européens. La campagne très active, usant d'arguments de toutes sortes, qu'ont menée en France les différentes sortes de partisans du "non", qu'ils soient d'extrême droite ou d'extrême gauche, a provoqué dans une grande partie de l'opinion française un déclin de l'idée, dans l'ensemble positive, que l'on se faisait jusqu'alors de l'Europe (comme dans la plupart des autres pays européens, à l'exception des Anglais qui y sont traditionnellement hostiles). La campagne des nationalistes de droite (contre l'immigration notamment) a profité de celle des mouvements de gauche contre le libéralisme économique. En refusant cette Constitution européenne, qui risquait selon eux d'accroître encore le chômage en France, ils en ont au fond rendu 
I'"Europe" responsable depuis des décennies.

La progressive extension de l'UE était jusqu'alors considérée comme un phénomène globalement positif. Certes on savait que les "aides européennes" allaient devoir être partagées entre un nombre croissant de pays à relativement bas niveau de vie, mais, comme dans le cas de l'Espagne, ceux-ci offriraient de nouveaux débouchés aux entreprises des pays européens à fort PNB. Mais cet argument des partisans du "oui" a été battu en brèche par ceux du non qui ont dénoncé le marasme économique de la France et de l'Allemagne, les pays "les plus européens" et bases de la monnaie européenne commune (que refuse toujours le Royaume-Uni).

Ce sont donc principalement des arguments économiques et sociaux inspirés par l'antilibéralisme et l'altermondialisme qui viennent de conduire à la mise en cause de l'idée européenne qui, jusqu'alors, avait été associée à celle d'une grande réussite dans les différents secteurs économiques. Les progrès de l'UE avaient été aussi justifiés par des raisons géopolitiques, qui étaient évoquées certes moins fréquemment mais de façon solennelle puisqu'ils permettent d'établir et de préserver la paix entre des États qui n'ont pas tous oublié leurs contentieux territoriaux. L'exemple de la tragédie yougoslave qui débute en 1991 avait en fait contribué à la signature des accords de Maastricht et ceux-ci ont fait passer la Communauté économique européenne à une UE dont la vocation à devenir un ensemble géopolitique était reconnue.

Cependant, en 1992 lors du référendum qui approuva en France (et de justesse) ces accords sur I'Europe, les rivalités de politique intérieure ne furent pas oubliées : une grande partie des électeurs de droite (malgré la prise de position de Jacques Chirac) votèrent non car le président et le gouvernement étaient alors de gauche, tout comme en mai 2005 une grande partie des électeurs de gauche (malgré une consultation préalable au sein du Parti socialiste) viennent de voter contre, car le président et le gouvernement sont de droite.

Cependant parmi les causes de la crise de l'idée européenne que vient de provoquer (ou de révéler) la campagne contre le projet de constitution européenne, les arguments géopolitiques ne manquent pas. Certains ont été plus ou moins implicites : l'opinion française, puisqu'elle a été invitée pour la première fois depuis 1992 à donner son avis sur l'UE, a manifesté au fond sa mauvaise humeur de se trouver devant le fait accompli d'une Europe, non plus à quinze mais à vingt-cinq États européens et bientôt à trente. L'opinion française qui avait massivement soutenu la politique de son gouvernement contre l'intervention américaine en Irak a été très choquée par le ralliement spectaculaire aux États-Unis des chefs de gouvernement de la "nouvelle Europe" selon la formule utilisée à la Maison Blanche et au Pentagone pour désigner les pays d'Europe de l'Est, nouveaux membres de l'UE et surtout de I'OTAN.

\section{La question de la Turquie}

C'est surtout la question éminemment géopolitique de l'adhésion de la Turquie à l'UE qui a été une des grandes raisons du succès du non au référendum sur la "constitution". Certes en principe les deux questions n'étaient pas liées, mais c'est au moment où en France il a commencé à être question de ce référendum qu'à "Bruxelles" le problème de la candidature de la Turquie a été posée de façon de plus en plus insistante. Cela remonte à une promesse qui aurait été faite au 
gouvernement d'Ankara, à l'époque de la guerre froide, lorsque l'armée turque était un des piliers du dispositif stratégique de l'OTAN face à l'Union soviétique. En 2002, la diplomatie américaine a ensuite fortement poussé à l'entrée de la Turquie dans l'UE pour convaincre les chefs de l'armée turque de participer à une intervention américaine en Irak. Mais, début 2003, les dirigeants turcs y ont renoncé et même, in extremis, se sont opposés à ce que des troupes américaines utilisent les bases de I'OTAN en Turquie pour envahir le nord de l'Irak, en se rendant compte que l'aide qu'elles allaient apporter aux Kurdes d'Irak favoriserait aussi les projets séparatistes des Kurdes de Turquie.

La question de l'adhésion de la Turquie à l'UE a provoqué en France un grand débat véritablement géopolitique. On y fait grand cas de la géographie ou de l'histoire : les uns soulignent que $3 \%$ seulement du territoire turc se trouve sur le continent européen, tel qu'il est délimité par les détroits du Bosphore et des Dardanelles, les autres contestent cette façon de voir les choses et rappellent que, durant des siècles, les Turcs ont joué un rôle important en Europe. En revanche, quelques-uns rappellent que ce fut en y opprimant et en massacrant nombre de Hongrois, de Serbes, de Bulgares et de Grecs. Jusqu'à ces dernières années, l'opinion grecque exprimait une forte opposition à une entrée de la Turquie dans l'UE, et en France l'on comptait sur cela pour qu'une candidature turque soit écartée. Mais aujourd'hui les milieux dirigeants grecs sont à ce propos singulièrement discrets (voir ci-après l'article de Michel Sivignon).

Un des grands arguments favorables à la candidature de la Turquie, tel que l'ont exposé le gouvernement français et quelques intellectuels de droite et de gauche (et spécialistes de géopolitique), est qu'il s'agit du seul pays musulman doté depuis trois quarts de siècle d'un État laïque et que l'Europe montrerait ainsi aux pays arabes qu'elle n'est pas un "club chrétien" (sic) et qu'elle accepterait d'autres pays musulmans, s'ils se ralliaient au principe de laïcité. $\mathrm{Si}$, dans les milieux populaires, le refus des Turcs se fonde sur des sentiments plus ou moins racistes et surtout sur la crainte des mouvements islamistes, les intellectuels, qui s'inquiètent de l'adhésion de la Turquie, estiment que sa "laïcité" n'est pas le sentiment de la majorité de la population, mais surtout la volonté de l'appareil d'État et de l'armée. Cette "laïcité" résulte de la volonté de Mustapha Kémal dans les années 1920 de marquer une rupture avec les Arabes qui venaient de trahir la Turquie, tout comme il fit interdire l'usage de l'alphabet arabe pour imposer aux Turcs l'alphabet latin et d'écrire de la gauche vers la droite (et non pas de la droite vers la gauche comme ils le faisaient jusqu'alors en utilisant l'alphabet arabe). On sait en effet que, durant la Première Guerre mondiale, en 1916, poussés par les Anglais, les Arabes du Moyen-Orient, qui dépendaient alors de l'empire ottoman, avaient "traîtreusement" attaqué l'armée turque, alors alliée de l'Allemagne et contribué à sa défaite.

De nos jours c'est encore principalement l'armée turque (tout au moins ses cadres), héritière de l'idéologie kémaliste, qui veille au respect de cette Constitution laïque. Or une des conditions majeures imposées par l'UE à la Turquie est de se "démocratiser", c'est-à-dire surtout de réduire considérablement les pouvoirs de l'armée, ce que les islamistes turcs approuvent tout à fait, puisque l'état-major est le principal défenseur de la laïcité dans la Constitution. L'opinion turque est en vérité assez partagée (voir ci-après l'article de Stéphane Yérasimos dont le nom grec n'exclut pas que ses liens avec la Turquie soient fort étroits). Le caractère pour le moment très modéré de l'islamisme en Turquie (hormis celui des Kurdes qui furent autrefois les seuls à s'opposer aux réformes de Mustafa Kémal) ne garantit pas que les islamistes radicaux n'y prennent tôt ou tard un rôle beaucoup plus important qu'ils n'ont actuellement. Ils sont déjà très influents parmi les Turcs émigrés en Allemagne et en France. 
L'autre grand argument contraire à l'adhésion de la Turquie est l'effectif de sa population Avec plus de 70 millions d'habitants et une croissance démographique qui est encore assez forte, elle serait dans vingt ans, avec plus de 90 millions d'habitants, l'État le plus peuplé de l'UE et elle disposerait donc dans les instances européennes du pourcentage de voix le plus élevé. L'entrée de la Turquie dans l'UE y accroîtrait encore l'importance et l'influence de l'immigration musulmane. De surcroît la Turquie fait partie du vaste ensemble des populations turcophones qui s'étend depuis l'Azerbaïdjan et l'ouest de l'Iran, l'Asie centrale et le sud de l'Oural (Tatarstan) jusqu'à l'ouest de la Chine (Ouïgours du Sinkiang). Une adhésion de la Turquie à l'UE aurait pour effet de faciliter l'immigration de turcophones venues de ces terres lointaines.

Le débat sur l'adhésion de la Turquie a d'ores et déjà pour effet de poser celui de pays qui en sont mitoyens, la Géorgie et l'Arménie. Certes ces États chrétiens sont situés au sud du Caucase, cette très grande montagne étant considérée traditionnellement comme la limite du continent européen entre la Caspienne et la mer Noire. Mais si la Turquie fait partie de l'UE, pourquoi pas eux ? Ils se réclament de l'idée en vérité fort répandue dans les pays européens (à l'exception de la France) que la majorité y soit catholique protestante ou orthodoxe et pour qui l'Europe est au fond un ensemble de diverses cultures chrétiennes plus ou moins laïcisées. Cette idée ne laisse pas indifférents, bien qu'ils soient de langue arabe, les chrétiens libanais qui vivent pour une grande part en Europe. Mais que penser d'Israël dont la grande majorité des populations est venue d'Europe ? Le rôle culturel considérable qu'elles y jouent depuis des siècles, les responsabilités historiques de l'Europe dans la Shoah font que les Européens ne peuvent se désintéresser du destin d'Israël. Est-ce un morceau d'Europe au Proche-Orient ? Pour le moment la grande majorité des Israéliens récusent l'UE qui prône l'accord avec les Palestiniens (voir ci après l'article de Frédéric Encel) et ils comptent principalement sur l'Amérique.

En vérité, c'est un paradoxe étonnant sinon même scandaleux que de discuter autant de l'entrée de la Turquie de culture musulmane dans l'UE, alors que l'on ne souffle mot de la Russie qui pourtant est indiscutablement de culture européenne. Mais la crise de l'idée européenne, provoquée ou révélée par les "non" français et néerlandais, fait que la question des nouveaux élargissements de I'"Europe" est pour le moment reportée à plus tard. II est probable que cette crise du consensus européen, qui souligne les désaccords des Anglais avec les Français et les Allemands, va faire apparaître les rivalités d'intérêt entre membres fondateurs et nouveaux adhérents, entre "grands pays" et "petits pays", etc.

Il va sans doute falloir attendre longtemps, peut-être plusieurs décennies, pour que l'UE s'élargisse de nouveau et pour qu'elle englobe l'ensemble des Européens, "de l'Atlantique à l'Oural" (comme disait de Gaulle) et surtout pour qu'elle devienne véritablement un ensemble politique. Mais cela peut aller bien plus vite si une grande menace islamiste se dressait brusquement sur sa façade sud, de la Méditerranée à l'Asie centrale, et si les Européens prenaient conscience qu'ils seront sans doute désormais seuls à devoir y faire face. En effet, aux États-Unis, l'opinion - de guerre lasse - souhaite de plus en plus qu'une solution honorable soit trouvée pour mettre fin à l'aventure irakienne, ce qui développe un processus comparable à celui qui avait mis fin en 1973 à leur aventure vietnamienne, deux ans avant la victoire communiste. 